\title{
How Coarse is Too Coarse in Electrically Large Reflectarray Smart Antennas?
}

\author{
A phase quantization case study at millimeter wave bands
}

\author{
Ghulam Ahmad, Tim W. C. Brown, Craig I. \\ Underwood \\ Faculty of Engineering and Physical Sciences (FEPS) \\ University of Surrey \\ Guildford, United Kingdom \\ \{g.ahmad, t.brown, c.underwood\}@surrey.ac.uk
}

\begin{abstract}
Millimeter wave (mm-wave) bands are becoming potentially attractive candidates for next generation communication systems. It is envisioned that high gain smart antennas will be one of the key enabling technologies for such systems. At mm-wave bands, where electrical size of an individual antenna becomes very small, the inclusion of a reconfigurable mechanism in the antenna becomes a great challenge due to real estate constraints. In these scenarios a designer has to decide on the number of bits in a phase shifter for antenna beam steering which will result in an optimum design. This contribution addresses the issue of phase quantization in mm-wave high gain reflectarray smart antennas to achieve an optimum performance. Implementing coarse phase quantization greatly reduces the complexity at $\mathbf{m m}$-wave bands. A case study is presented to highlight the effects of coarse phase quantization using various numbers of bits.
\end{abstract}

Keywords-Smart antennas, reflectarray, phase quantization, high gain antennas, millimeter waves

\section{INTRODUCTION}

A quest for higher data transfer rates resulting in low latencies to enable futuristic applications supported by next generation communication systems has initiated an intensive research in the field of millimeter wave (mm-wave) bands. High gain mm-wave smart antennas are one of the key enabling technologies for such communication systems. A smart antenna needs to incorporate a reconfiguration mechanism to steer its radiated beam towards the intended direction. At mm-wave bands where real estate becomes a constraint in antennas, it is necessary to simplify the reconfiguration mechanism while preserving an acceptable antenna performance [1].

Reconfiguration of the radiation pattern in a simplified form is usually achieved by controlling the excitation phases of the antenna elements in an array. To attain high gain, one may need to use thousands of antenna elements [2]. To implement such a high number of radio frequency (RF) chains is impractical. Therefore, it is preferable to implement reconfiguration mechanism in the antenna itself at the RF plane comprising of antenna array elements. Reflectarray antennas are one of the compelling candidates for such implementations.

This research was jointly sponsored by Surrey Space Center; University of Surrey and National Physical Laboratory under the project code SSC_NPL_KB4545 as a part of research on mm-wave reflectarray antennas. The work of T. H. Loh was supported by the $2013-2016$ and $2016-2017$ Quantum, Electromagnetics and Time program of the National Measurement Office, an Executive Agency of the U.K. Department for Business, Energy and Industrial Strategy, under Projects 116774 and 119575.

\author{
Tian Hong Loh \\ Time, Quantum and Electromagnetics Division \\ National Physical Laboratory (NPL) \\ Teddington, United Kingdom \\ tian.loh@npl.co.uk
}

Reflectarrays benefit from spatial illumination of its element (unit cells) from a feeding source therefore, avoiding the losses in array feeding mechanisms and preserving a single RF chain [3]. It enables the flexibility to control the reflection phase from each unit cell to direct the array radiated beam in the desired direction.

Implementations for continuous phase control or a higher number of phase quantization bits are not practically feasible [4] due to either non-availability of electronic devices or their inclusion in a very small physical size dictated by antenna electrical design. Due to a large number of elements in a reflectarray, the reconfiguration mechanism in each unit cell needs drastic simplifications to realize a practically implementable design [5]. In this contribution we have presented the effects on antenna performance resulting from such simplifications. We considered various number of phase quantization bits and studied the resulting effects on radiation pattern, directivity loss, scan range, and bandwidth for a smart high gain reflectarray antenna at $60 \mathrm{GHz}$.

Section II presents the reflectarray structure and its performance for various number of phase quantization bits. Section III concludes the contribution.

\section{REFLECTARRAY AND PHASE QUANTIZATION}

A reflectarray operates on the principle that a constant phase of the reflected field is achieved in a plane normal to the direction of the desired antenna main beam. At the center point of each unit cell the required phase is calculated as [3]:

$$
\frac{2 \pi}{\lambda_{0}}\left(r_{m n}-\vec{R}_{m n} \cdot \hat{r}\right)-\Delta \Phi_{m n}=2 \pi N
$$

where $\lambda_{0}$ is free space wavelength, $\vec{r}_{m n}$ is the position vector of $m n^{\text {th }}$ element/unit cell relative to $(0,0, F), F$ is the focal length, $\vec{R}_{m n}$ is the position vector of $m n^{\text {th }}$ element relative to $(0,0,0)$, $\hat{r}_{b}$ is the direction vector of the desired pencil beam, $N=$ $1,2,3 \ldots$, and $\Delta \Phi_{m n}$ is the phase shift introduced by $m n^{\text {th }}$ unit cell of reflect array to its reflected field relative to the incident field. For geometry illustration a typical printed microstrip based reflectarray structure is partly shown in Fig. 1. It consists of a large number of unit cells containing reflecting patches on a grounded substrate. These unit cells are spatially excited by a suitable feed horn. 


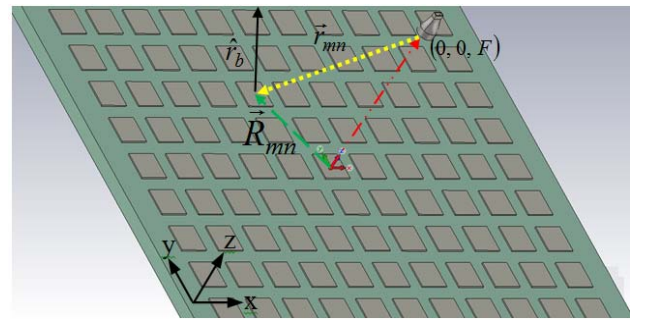

Fig. 1. Printed microstrip reflectarray on a grounded substrate

In a smart reflectarray the passive unit cells shown in Fig. 1 are replaced by unit cells which implement the phase control mechanism to provide the required reflection phase from its location in the reflectarray. In addition to the accommodation of multiple control elements the DC biasing for an increased number of unit cells further complicates the design and results in a significant performance loss. It is preferable to have as few as possible DC bias lines per unit cell to simplify the design at mm-waves.

Table 1 lists the loss in directivity for various bits of phase shifter in comparison to continuous phase shift for a center fed $20 \lambda$ square reflectarray of square patches with $0.54 \lambda$ unit cell spacing and $15 \lambda$ focal length. A three bit phase quantization practically results in negligible directivity loss. Therefore, it is taken as a reference to normalize the directivity values for CST simulations. Here it can be seen that directivity loss increases when implementing coarse phase quantization; however doing so simplifies the DC biasing and real estate issues as it requires less number of discrete electronic elements. The difference in Matlab ${ }^{\circledR}$ calculated and CST simulated values is due to the fact that reflection phase was calculated using (1); however, CST simulations consider more practical implementation of small scattering of reflection phase around the exact desired value due to array behavior and incident angle dependence. Moreover, judiciously selected reflecting patch dimensions have shown a slight decrease in loss. A single bit implementation would result in an excessive loss in directivity which may not be practically feasible for certain situations. However, by just increasing the phase states to three i.e. implementing a 1.5 bit phase shifter considerably reduces the loss in directivity to a practically acceptable solution. It is possible to achieve three phase states while still maintaining the DC biasing complexity of a one bit phase shifter.

Table 2 lists the simulated bandwidth and maximum scan range as simulated in CST and Matlab ${ }^{\circledR}$ respectively. Although the grating free scan range was $55^{\circ}$, however the beam can be scanned to a maximum of approximately $77^{\circ}$ at the cost of reduced gain due to grating lobes. A similar number for maximum scan range was observed through CST simulations of current study and $0.5 \lambda$ spaced unit cells reflectarray. Mainly, specular reflections from unit cells limit the scan range and no further improvements were seen. The bandwidth improves as the phase quantization become coarse due to the fact that it is more tolerant to phase changes because of bigger phase steps.

The $3 \mathrm{~dB}$ bandwidth response generated by CST simulations is shown in Fig. 2 where all the arrays were primarily designed at $60 \mathrm{GHz}$. Due to the selection of unit cell patches to produce the desired reflection phase, a shift in the reflectarray frequency bandwidth was observed; the coarser the quantization, higher is the shift in operating band. This is not a
Table 1: Directivity loss w.r.t. phase quantization bits

\begin{tabular}{|l|c|c|}
\hline \multirow{2}{*}{$\begin{array}{c}\text { Number of phase } \\
\text { quantization bits }\end{array}$} & \multicolumn{2}{|c|}{ Loss in Directivity (dB) } \\
\cline { 2 - 3 } & Matlab $^{\circledR}$ code & CST Simulation \\
\hline Continuous phase shift & 0 & --- \\
\hline 3 bits (8 phase states) & 0.22 & --- \\
\hline 2 bits (4 phase states) & 0.99 & 0.7 \\
\hline 1.5 bits (3 phase states) & 1.66 & 0.9 \\
\hline 1 bit (two phase states) & 3.92 & 2.7 \\
\hline
\end{tabular}

Table 2: Bandwidth and maximum scan angle of reflectarrays

\begin{tabular}{|l|c|c|}
\hline \multicolumn{1}{|c|}{$\begin{array}{c}\text { Number of phase } \\
\text { quantization bits }\end{array}$} & Bandwidth & Max. scan range \\
\hline Continuous phase & -- & $76.8^{\circ}$ \\
\hline 3 bits (8 phase states) & $8.8 \mathrm{GHz}$ & $76.8^{\circ}$ \\
\hline 2 bits (4 phase states) & $9.0 \mathrm{GHz}$ & $76.8^{\circ}$ \\
\hline 1.5 bits (3 phase states) & $10 \mathrm{GHz}$ & $76.5^{\circ}$ \\
\hline 1 bit (two phase states) & $10.2 \mathrm{GHz}$ & $76.7^{\circ}$ \\
\hline
\end{tabular}

drawback; the reflectarray can be made to operate at the desired band by slightly changing the patch dimensions.

When the radiated beam is scanned away from the boresight, it results in a directivity loss. Matlab ${ }^{\circledR}$ code was used to generate the scan loss curves as shown in Fig. 3 for various beam pointing angles. A consistent degradation of directivity can be observed for each curve shown including continuous and phase quantized reflectarray. In Fig. 4, the directivity loss for quantized phase reflectarrays relative to the continuous phase reflectarray at various beam pointing angles; known as differential scan loss, is shown. Almost constant differential scan loss in each curve shown indicates the beam scanning loss is almost independent of the phase quantization.

As the reflectarray contains a significantly high number of reflecting unit cells, therefore, the beam pointing error; when phase quantization takes place, is not significantly high over the entire scan range in comparison to continuous phase reflectarray. It is shown in Fig. 5; where achieved pointing angles are plotted with respect to the desired pointing angles. The achieved pointing angle curves saturate near $77^{\circ}$ in all the cases including continuous phase reflectarray and the beam point error increases without any significant further improvement in achieved pointing angle.

Fig. 6 displays co-polar radiation patterns of phase quantized reflectarrays generated by CST. Three bit phase quantized reflectarray directivity was used to normalize the directivity of other phase quantized reflectarrays. There is a loss in directivity at boresight due to phase quantization. In general the first sidelobe is around $20 \mathrm{~dB}$ down the boresight directivity. The far out sidelobes are well below the boresight directivity for most of the practical purposes.

The cross-polar radiation response is not significantly affected due to phase quantization as observed in CST simulations. In all the cases with a feed horn having around 40 $\mathrm{dB}$ cross polarization discrimination, we were able to achieve better than $35 \mathrm{~dB}$ cross polarization discrimination at reflectarray level. 


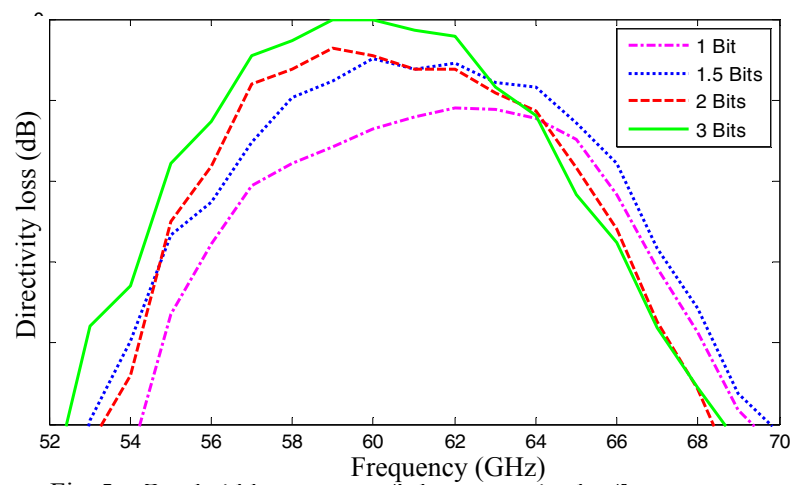

Fig. 2. Bandwidth response of phase quantized retlectarrays

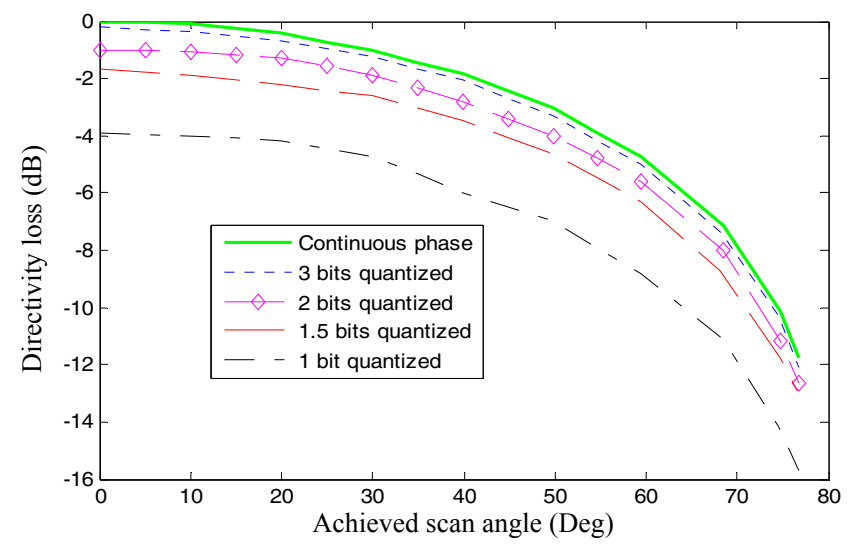

Fig. 3. Beam scan angle and directivity loss in reflectarrays

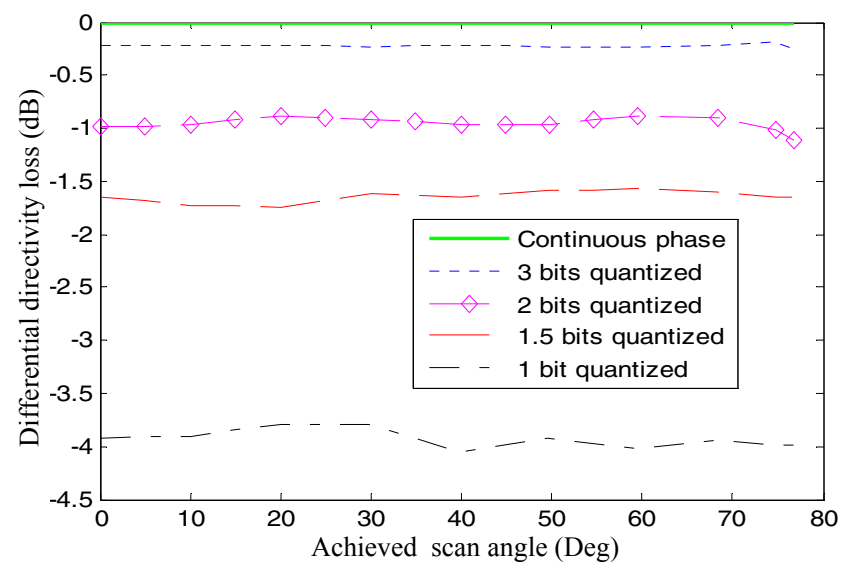

Fig. 4. Differential beam scanning loss in reflectarrays

\section{CONCLUSION}

This study presented a comparison of phase quantized reflectarrays w.r.t. continuous phase reflectarray at $60 \mathrm{GHz}$. With phase quantization, a loss in boresight directivity is observed which increases as the phase quantization becomes more coarse. A severely coarse phase quantization of one bit with two phase states produces the highest calculated loss of $3.9 \mathrm{~dB}$. However, with a suitable choice of reflecting patches this can be reduced to less than $3 \mathrm{~dB}$ as shown in CST simulations. A 1.5 bit phase quantized reflectarray exhibits the most desirable performance tradeoff at mm-wave bands due to significantly less complex DC biasing network required for its implementation. The higher phase quantization bits result in better directivity; however may not be optimum when implementation complexity is considered at mm-wave bands

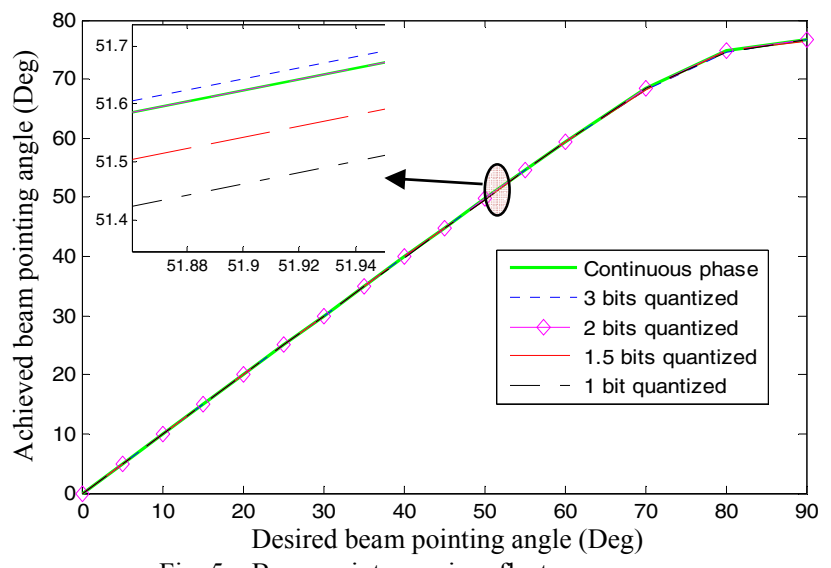

Fig. 5. Beam point error in reflectarrays

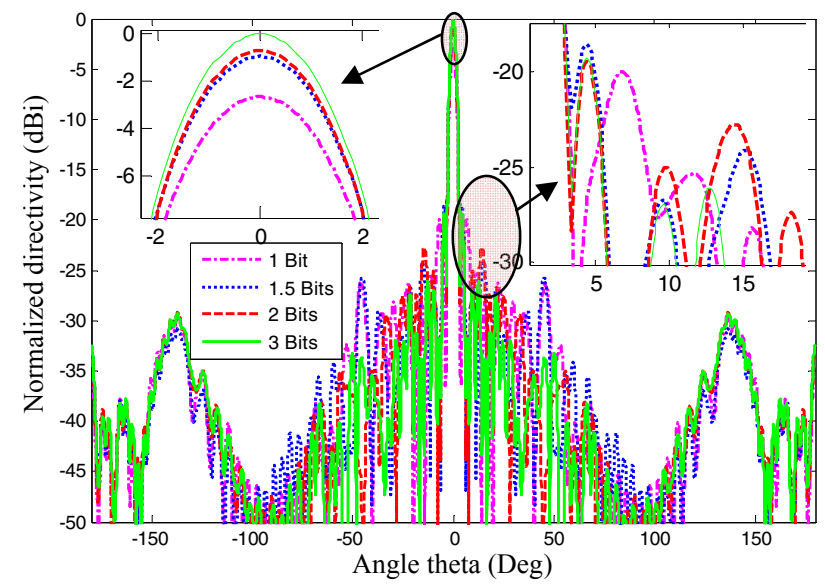

Fig. 6. Radiation pattern of phase quantized reflectarrays

where real estate is a potential issue. A smooth and consistent directivity degradation due to beam scanning was observed for all the cases of phase quantization. The beam pointing error is minimal and a reliable beam scanning can be achieved in all the cases of phase quantization. In radiation pattern the first sidelobe levels are around $20 \mathrm{~dB}$ down in all the cases. The cross polarization discrimination was observed not being a strong function of phase quantization. Results presented in this study shall produce a significant impact in the choice of antennas for next generation communication systems.

\section{REFERENCES}

[1] J. Song, J. Wang, K. Peng, C. Pan, and Z. Yang, "Quantization error reduction for the phased array with 2-bit phase shifter," Wireless personal communications, vol. 52, pp. 29-41, 2010 .

[2] H. Kamoda, T. Iwasaki, J. Tsumochi, T. Kuki, and O. Hashimoto, "60$\mathrm{GHz}$ electronically reconfigurable large reflectarray using single-bit phase shifters," Antennas and Propagation, IEEE Transactions on, vol. 59, pp. 2524-2531, 2011.

[3] S. V. Hum and J. Perruisseau-Carrier, "Reconfigurable reflectarrays and array lenses for dynamic antenna beam control: A review," Antennas and Propagation, IEEE Transactions on, vol. 62, pp. 183-198, 2014.

[4] B. Wu, A. Sutinjo, M. E. Potter, and M. Okoniewski, "On the selection of the number of bits to control a dynamic digital MEMS reflectarray," Antennas and Wireless Propagation Letters, IEEE, vol. 7, pp. 183-186, 2008.

[5] M.-T. Zhang, S. Gao, Y.-C. Jiao, J.-X. Wan, B.-N. Tian, C.-B. Wu, and A.-J. Farrall, "Design of Novel Reconfigurable Reflectarrays With Single-Bit Phase Resolution for Ku-Band Satellite Antenna Applications," IEEE Transactions on Antennas and Propagation, vol. 64, pp. 1634-1641, 2016. 